\title{
Study on the variation law of atmospheric quality and the mutual influence between regions
}

\author{
Zijun Song \\ School of Electrical \& Electronic Engineering, North China Electric Power University, Hebei 071000, \\ China
}

18233274031@163.com

Keywords: time series ARIMA Partial differential diffusion models UTM projection

\begin{abstract}
In this paper, to establish the mathematical model of Beijing Tianjin Hebei region air quality change existing data analysis and processing analysis, air quality in the region between the mutual influence relations. Finally, according to the analysis results, this paper puts forward the methods and measures of air pollution. Time series model of air quality change in different cities. The ARIMA model is not strong enough to use the wave period. Based on the diffusion principle of air pollutants, the partial differential attenuation dispersion model is established, and the simulation results are very good.
\end{abstract}

\section{Introduction}

Due to the increasingly serious problem of haze, the public began to focus more and more fine particulate matter (PM10) and respirable particulate matter (PM2.5). With the convening of the APEC meeting, "APEC" Blue become a network of hot words, air quality will become a reality and strategic problems that restrict the development of the region to be solved.

By analyzing and processing of existing data, a mathematical model of air quality of Beijing, Tianjin region change, air quality analysis of mutual influence relations between the region and eventually propose a method to control air pollution and measures based on results of the analysis.

\section{2 model assumptions}

1. Air Quality Index (AQI) is the same, the same degree of contamination, without considering the specific physical and chemical properties of different pollutants.

2. The index does not consider the air with the elevation of the impact that the concentration of each height at the same ground surface.

3. Under no abnormal weather, the indicators are considered diffusion in natural circumstances.

\section{Establishment and solution of the model}

\subsection{Beijing, Tianjin and regional air quality model change}

By observing and drawing 13 urban air quality index curve in January and March, and found the same trend in cities and have been to the data is arranged in chronological order, changes over time and interrelated sequence of data is seasonal variation coupled with irregular movement, using a mathematical model ARIMA time series.

Use MATLAB data for each city AQI do autocorrelation and partial autocorrelation function diagram, and solution time series model binding AIC test, Hebei Baoding as an example:

Autocorrelation and partial autocorrelation function diagram Baoding truncation phenomenon does not appear, $\mathrm{p}$ and $\mathrm{q}$ is not 0 , the use of AIC parameters determined by MATLAB programming available $\mathrm{p}=6, \mathrm{q}=6$, the smallest $\mathrm{AIC}=8.30$

Baoding the Air Quality Model can be expressed as: 


$$
\begin{aligned}
& \mathrm{X}(t)=-0.9003 X_{t-1}+0.6517 X_{t-2}-0.2438 X_{t-3}-0.4406 X_{t-4}+0.328 X_{t-5}-0.3698 X_{t-6}+ \\
& \varepsilon_{t}-0.5967 \varepsilon_{t-1}+0.7450 \varepsilon_{t-2}-0.0029 \varepsilon_{t-3}-0.7063 \varepsilon_{t-4}+0.6065 \varepsilon_{t-5}-0.9543 \varepsilon_{t-6}
\end{aligned}
$$

Use EXCEL to draw a March AQI fitting renderings shown in Fig. 1:

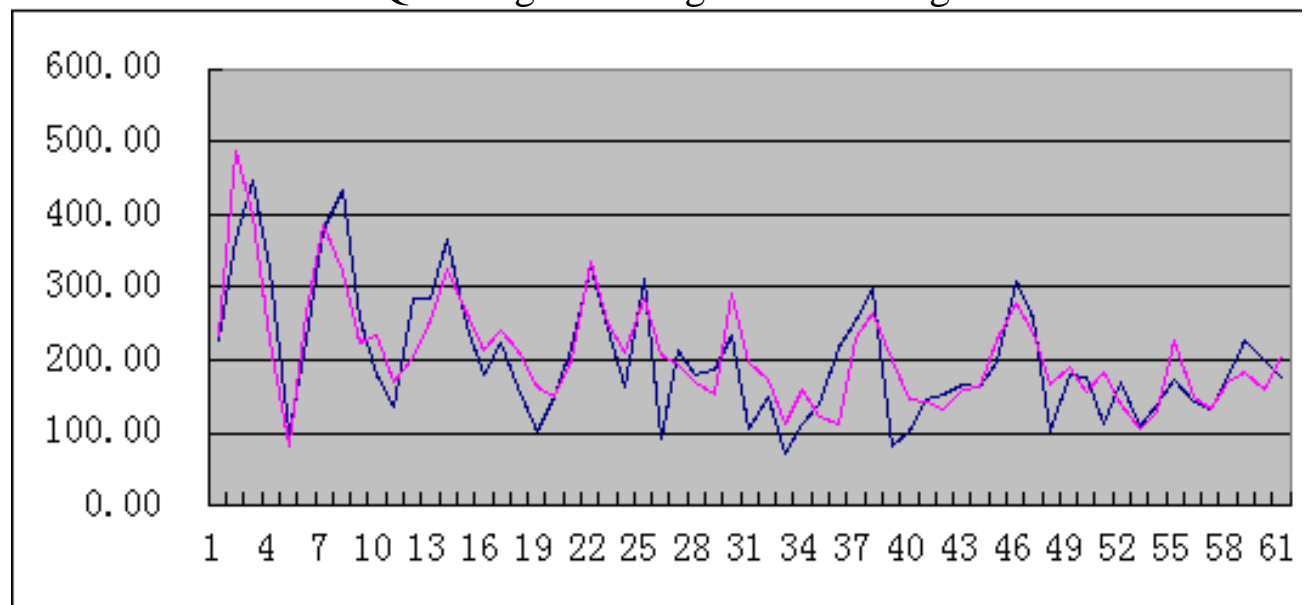

Fig. 1 Baoding in January and March AQI fitting renderings

ARMA model fitting results show that:

a whole. The simulation results better, in addition to fitting error of some large peak point, the fitted value is consistent with the measured values. Forecast effect between qualified and better explain the established time series model can objectively reflect the actual. To reduce the fitting error, the effect of improving forecasting model, we implanted Markov forecasting model.

Step using the relative error probability transition matrix of time-series forecasting models were corrected Markov, the second was the fit value and a quadratic fit with the measured values.

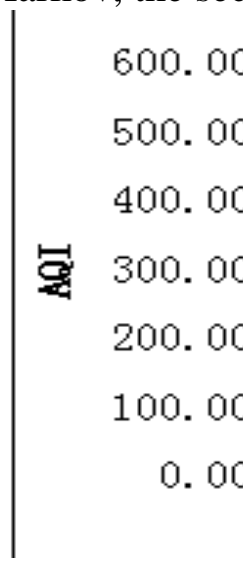

The results show that :

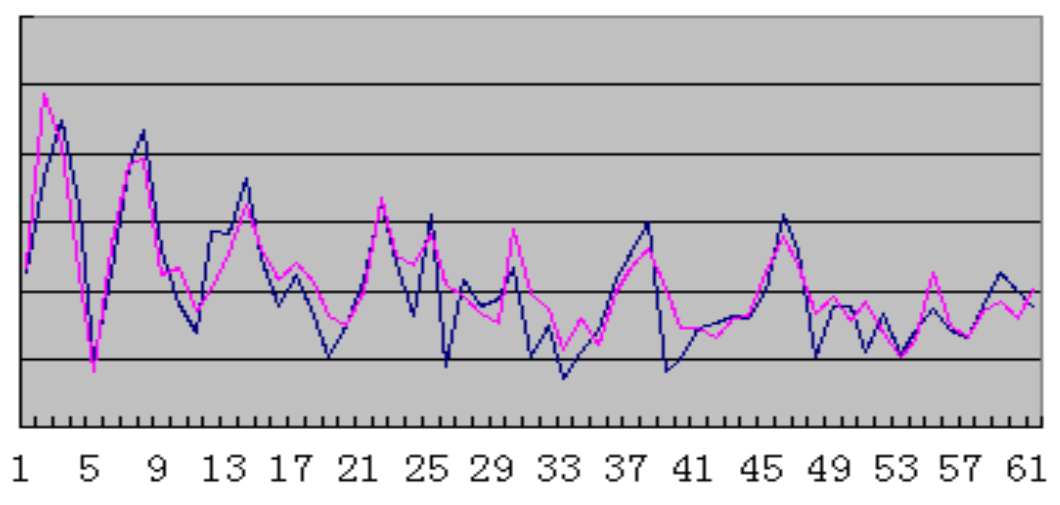

Fig. 2 Baoding Markov Improvement

prediction and measured values based on time series analysis Markov basically the same, the peak of the fitting error is significantly less than a single time series model error.

\section{2 interaction between regional air quality relationship analysis}

Through the establishment of diffusion model, analysis of regional interaction relationship between air quality.

\subsection{1 diffusion partial differential attenuation model}

Taking into account the actual situation, find a greater impact Gaussian model in the wind direction, and the coordinates of the given point is not the real source of transmission, it is unreasonable Gaussian diffusion model; partial differential model, although not considered wind direction and speed, but you can parameter fitting to determine wind direction and speed. Therefore, this final choice of partial differential model simulation. 


\subsubsection{Model Solution}

Solving the model here is the solution parameters. Therefore, the data obtained by the approximate correlation coefficients, thereby forming a final diffusion decay model.

Solutions obtained by the MATLAB:

$$
\alpha=-10.7 \times 10^{-12} \quad \beta=-30.7 \times 10^{-12} \quad \varepsilon=5.59
$$

As can be seen from the residual plots, in addition to an abnormal data, the remaining data from the residuals are close to zero, and the residual confidence interval contains zero, indicating that the regression model can better match the original data.

Regression model $K=-10.7 \times 10^{-12} X-30.7 \times 10^{-12} Y+5.59$ was established.

\subsubsection{Model Checking}

The accuracy of the model for, as an example to AQI, the specific reference to the measured data for analysis.

Here Select AQI data January 10 through inspection data, windless day, so do not consider the impact of wind speed and direction, calculated in accordance with this model, taking the highest concentration of point AQI January 10 most sources can be simulated 1 graphics March 11 are as follows:

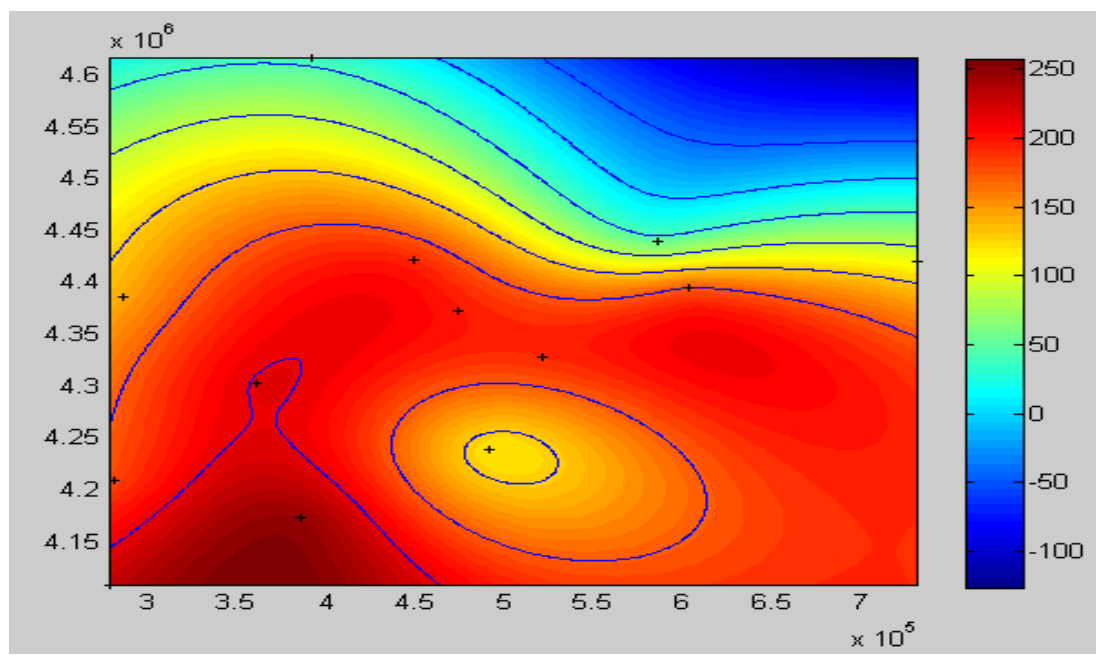

Fig. 3 January 11 AQI Rendering



Fig. 4 January 11 AQI actual diagram

This figure compared with the actual distribution, relatively small differences were found, the cause of the difference may be:

1) actual diffusion is very complex, many factors such as heat island effect, local circulation as a result of diffusion does not work as the model situation.

2) model building process, not considering temperature effect. 


\section{Conclusion}

Firstly, by looking at the data, the establishment of ARIMA (p, d, q) time series model, better fitting the AQI daily average of January and March regions of Beijing, Tianjin, and through the Markov model the model is improved so that better data fitting results.

In order to analyze the interaction between pollutants in various regions of the relationship, but also compared the mechanism of the Gaussian model of atmospheric diffusion and partial differential decay diffusion model, final selection of the model may reflect partial differential attenuation.

In addition, the spread of air pollutants but also by altitude, distance, density, wind speed, wind direction, and this model had been amended to improve the applicability of partial differential model. Secondly, in the calculation of air pollution models to simulate the graphics to give good results, relevant departments can use this model to PM2.5, and PM10 forecasting.

\section{References}

[1] approach missing values, http: //www.itongji.cn/article/100311B2012.html

[2] Information on http: //wenku.baidu.com/view/82acle4633687e2 1af45a930.html

[3] HJ 633-2012, Ambient Air Quality Index (AQI) technical requirements (for Trial Implementation) [S]

[4] Beijing Municipal Environmental Protection Monitoring Center.

[5] Meng, the main air pollution source inventory survey and analytical studies - in Shanxi source Case Study [D] Nanjing Information Engineering University .2006, 24-24.

[6] Qian Zhong, groundwater resources evaluation in a time series of precipitation Markov model [D]. Department of Earth Sciences

[7] When Qinghua, Comparison of two wind power prediction model [D]. Shandong Province Power Company. 